\title{
Lactobacillus gasseri SBT2055 suppresses fatty acid release through enlargement of fat emulsion size in vitro and promotes fecal fat excretion in healthy Japanese subjects
}

\author{
Akihiro Ogawa ${ }^{1 *}$, Toshiya Kobayashi ${ }^{2}$, Fumihiko Sakai ${ }^{1}$, Yukio Kadooka ${ }^{1}$ and Yoshihiro Kawasaki ${ }^{1}$
}

\begin{abstract}
Background: Lactobacillus gasseri SBT2055 (LG2055) has been shown to prevent abdominal adiposity, and suppression of lipid absorption is considered a possible mechanism, detail of which, however, are poorly understood. In the present study, we evaluated the effects of LG2055 on fat hydrolysis by determining pancreatic lipase activity and fat emulsion properties in vitro. We also examined whether LG2055 influences fecal fat excretion in humans.

Methods: Pancreatic lipase activity was investigated in vitro using an artificially prepared fat emulsion and 4-methylumbelliferyl oleate (4-MUO) as substrates. The concentrations of free fatty acids and 4-methylumbelliferone were quantified. Fat emulsion droplet size was measured using a particle size analyzer. The clinical study was performed as a double-blind, randomized, placebo-controlled trial. Subjects consumed $100 \mathrm{~g}$ of fermented milk (FM)/d, either with or without LG2055 supplementation, for seven days. Fecal samples were collected during three-day pre-observational and FM intake periods and fecal fat levels were determined.
\end{abstract}

Results: LG2055 dose-dependently suppressed lipase activity in the fat emulsion assay but not in the 4-MUO assay. LG2055 dose-dependently increased fat emulsion droplet size. The effects of LG2055 on lipase activity and fat emulsion properties were increased compared with four other tested strains (Lactobacillus gasseri SBT0317, Lactobacillus gasseri JCM1131 ${ }^{\top}$, Lactobacillus. delbrueckii subsp. bulgaricus JCM1002 ${ }^{\top}$ and Streptococcus thermophilus ATCC19258 ${ }^{\top}$ ). In our clinical study, fecal fat level after FM intake was significantly increased compared with that observed before FM intake in the LG2055-containing active FM group but not the control FM group lacking LG2055.

Conclusions: LG2055 increased fat emulsion droplet size, resulting in the suppression of lipase-mediated fat hydrolysis. The influence of LG2055 on the physicochemical properties of fat emulsion provides a mechanism for the probiotic-mediated suppression of lipid absorption and promotion of fecal fat excretion in humans.

Trial registration: UMIN000015772

Keywords: Probiotics, Lactobacillus gasseri SBT2055, Lipid absorption, Lipase, Fat emulsion, Droplet size, Fecal fat excretion

\footnotetext{
* Correspondence: akihiro-ogawa@meg-snow.com

${ }^{1}$ Milk Science Research Institute, Megmilk Snow Brand Co. Ltd., 1-1-2

Minamidai, Kawagoe, Saitama 350-1165, Japan

Full list of author information is available at the end of the article
} 


\section{Background}

Lactobacillus gasseri SBT2055 (LG2055), a probiotic lactic acid bacterium originating in the human intestine [1,2], has an ability to improve the intestinal environment [3] and exerts anti-obesity effects in rats [4-6] and mice [7]. Our previous clinical studies in Japanese adults with overweight exhibited a significantly decreased visceral fat area, body weight, body mass index (BMI), and waist and hip circumferences following consumption of fermented milk containing LG2055 at $200 \mathrm{~g} / \mathrm{d}$ for 12 weeks [8,9].

Suppression of lipid absorption in the small intestine has been proposed as a potential mechanism for the anti-obesity effects of LG2055. Hamad et al. evaluated lymphatic lipid content in rats with permanent cannulation of the thoracic duct [4]. They demonstrated that rats fed a diet containing fermented skim milk supplemented with LG2055 showed a lower maximal rate of lymphatic lipid absorption compared with rats fed a diet containing non-fermented skim milk; these findings were supported by the observation of increased fecal fatty acid excretion [4]. Furthermore, our recent study showed that Japanese hypertriacylglycerolemic subjects who consumed fermented milk containing LG2055 at $200 \mathrm{~g} / \mathrm{d}$ for 4 weeks demonstrated significantly decreased postprandial serum lipid concentrations after the intake of oral fat-loading test meals [10]. Serum lipid concentrations are influenced by not only lipid absorption via diet but also lipid metabolism. Therefore, estimation of fecal lipid excretion is a more direct index to examine dietary lipid absorption.

Dietary lipid digestion undergoes several complex processes prior to mucosal absorption in the small intestine. Pancreatic lipase is a key enzyme for lipid absorption because the majority of lipolysis is carried out in the duodenum by pancreatic lipase that is secreted from the pancreas and hydrolyzes dietary lipid-derived triacylglycerol into glycerol and fatty acids [11]. Dietary triacylglycerol, the major source of dietary fat, is not directly absorbed in the intestine unless it has been hydrolyzed by pancreatic lipase. Therefore, suppression of lipase-mediated fat hydrolysis is an effective approach for suppression of dietary triacylglycerol absorption [12].

Suppression of lipase-mediated fat hydrolysis is mainly associated with two mechanisms: the first is direct enzymatic inhibition by an inhibitor like orlistat, which is a potent pancreatic lipase inhibitor used as a pharmaceutical agent for the management of obesity [13]; the second is associated with modification of fat emulsion properties. Fat emulsion interface properties, including droplet size and specific surface area, influence the effects of lipasemediated fat hydrolysis on dietary fat absorption [14]. As fat emulsion particle diameter increases, specific surface area decreases. Thus, lipase-mediated fat hydrolysis is sensitive to fat emulsion size [15], and the change in fat emulsion droplet size is critical for modification of fat digestion and absorption. However, the mechanism of LG2055-mediated suppression of lipid absorption is unclear.

In this study, we investigated the mechanism associated with suppression of lipid absorption by the probiotic bacterium LG2055. We determined LG2055 effects on pancreatic lipase-mediated hydrolysis of an artificial fat emulsion or synthetic substrate (lipase activity) and measured fat emulsion droplet size in vitro using a simple oil-in-water emulsion as a physiological model. We also examined whether intake of LG2055 altered fecal fat excretion in healthy Japanese subjects.

\section{Methods \\ In vitro study \\ Materials}

Triolein, taurocholic acid, pancreatic lipase (type VI-S, from porcine pancreas), orlistat, and 4-methylumbelliferyl oleate (4-MUO) were purchased from Sigma-Aldrich Co., Ltd. (St. Louis, MO, USA). N-Tris (hydroxymethyl) methyl-2-aminoethanesulfonic acid (TES) was purchased from Dojindo Laboratories (Kumamoto, Japan). Lecithin from egg, NEFA C Test Wako, and catechin mixture from green tea (product number 032-18231) were purchased from Wako Pure Chemical Industries Co., Ltd. (Osaka, Japan).

\section{Preparation of LG2055 and other bacteria}

Lactobacillus gasseri SBT2055 (LG2055), a bacterial strain derived from a fecal specimen of a healthy adult originally isolated by Fujiwara et al. [3] was deposited in the International Patent Organism Depositary, National Institute of Advanced Industrial Science and Technology (Tsukuba, Ibaraki 305-8566, Japan). Lactobacillus gasseri SBT0317 (LG0317) was isolated from a dairy product and stocked at Megmilk Snow Brand Co. Ltd. [16]. Lactobacillus gasseri JCM1131 ${ }^{\mathrm{T}}$ (LG1131T) and Lactobacillus delbrueckii subspecies (subsp.) bulgaricus JCM1002 ${ }^{\mathrm{T}}$ (LB1002T) were obtained from the Japan Collection of Microorganisms. Streptococcus thermophilus ATCC19258 ${ }^{\mathrm{T}}$ (ST19258T) was obtained from the American Type Culture Collection (Manassas, VA, USA). Each strain was grown at $37^{\circ} \mathrm{C}$ for $16 \mathrm{~h}$ in de Man, Rogosa and Sharpe (MRS) broth (Becton-Dickinson and Company, MD, USA). Harvested cells of each strain were washed twice with saline and once with sterilized water, then lyophilized using a freeze dryer (FDU-2200, Tokyo Rikakikai Co., Ltd., Tokyo, Japan).

\section{Measurement of pancreatic lipase activity}

Pancreatic lipase activity using fat emulsion as a substrate was determined using the method described by Han et al. [17]. A fat emulsion was prepared by 
sonication of lipid suspensions composed of triolein $(80 \mathrm{mg})$, lecithin $(10 \mathrm{mg})$, and taurocholic acid $(5 \mathrm{mg})$ in $9 \mathrm{ml}$ of TES buffer (0.1 M TES, $0.1 \mathrm{M} \mathrm{NaCl}$, pH 7.0) for 25 min. Bacterial cells of each strain were suspended in TES buffer. For the enzyme reaction, $100 \mu \mathrm{l}$ of a bacterial cell suspension or orlistat solution and $50 \mu \mathrm{l}$ of pancreatic lipase (10 units) were added to $100 \mu \mathrm{l}$ of sonicated substrate suspension (fat emulsion) in a total volume of $250 \mu \mathrm{l}$, and the reaction mixture was incubated at $37^{\circ} \mathrm{C}$ for $30 \mathrm{~min}$. After completion of the reaction, the solution was heated in a boiling water bath for 2 min for enzyme inactivation. The blank of each sample was heated in a boiling water bath for 2 min immediately following addition of the enzyme solution for inactivation. The concentration of released fatty acids was measured using a NEFA $C$ Test Wako. To examine dose-dependency, LG2055 suspensions were prepared at final concentrations ranging from $1-100 \mu \mathrm{g} / \mathrm{ml}$. The effects of five bacterial strains (LG2055, LG1131T, LG0317, LB1002T and ST19258T) were compared using preparations at final concentrations of $100 \mu \mathrm{g} / \mathrm{ml}$. Pancreatic lipase activity of each sample was calculated using fatty acid production in the absence of sample as $100 \%$. Lipase activity using $4-\mathrm{MUO}$ as a substrate [18] was determined using the method described by Nakai et al. [19]. LG2055 cells were suspended in distilled water. Twenty-five microliters of a LG2055 suspension or an orlistat solution and $50 \mu \mathrm{l}$ of a $0.1 \mathrm{mM} 4-\mathrm{MUO}$ solution dissolved in Tris buffer consisting of $13 \mathrm{mM}$ Tris- $\mathrm{HCl}, 150 \mathrm{mM} \mathrm{NaCl}$, and $1.3 \mathrm{mM} \mathrm{CaCl}_{2}(\mathrm{pH} 8.0)$ were mixed in the well of a microtiter plate, and $25 \mu \mathrm{l}$ of the lipase solution $(50 \mathrm{U} / \mathrm{ml})$ in Tris buffer was then added to initiate the enzyme reaction. After incubation at $25^{\circ} \mathrm{C}$ for $30 \mathrm{~min}, 100 \mu \mathrm{l}$ of $0.1 \mathrm{M}$ sodium citrate $(\mathrm{pH} 4.2)$ was added to terminate the reaction. The amount of 4-methylumbelliferone released following the lipase reaction was measured using a fluorometrical microplate reader (Varioskan $^{\text {Tn }}$ Flash, Thermo Fisher Scientific, Inc., MA, USA) at an excitation wavelength of $327 \mathrm{~nm}$ and an emission wavelength of $449 \mathrm{~nm}$. The LG2055 suspensions and orlistat solutions were prepared at final concentrations ranging from 1-100 $\mu \mathrm{g} /$ $\mathrm{ml}$, and $0.1-100 \mu \mathrm{g} / \mathrm{ml}$, respectively. Pancreatic lipase activity of each sample was calculated using 4methylumbelliferone production in the absence of sample as $100 \%$.

\section{Measurement of particle size of fat emulsion}

LG2055 was suspended in $1.2 \mathrm{ml}$ of TES buffer and added to $8 \mathrm{ml}$ of the fat emulsion preparation. After addition of LG2055, the $\mathrm{pH}$ of the suspension was adjusted to 7.5 using $\mathrm{NaHCO}_{3}$, to reflect the in vivo condition, in which pancreatic juice containing alkaline sodium (sodium bicarbonate $\left(\mathrm{NaHCO}_{3}\right)$ ) is secreted into the small intestine for maintenance of a neutral $\mathrm{pH}$. TES buffer was used as a negative control, whereas a catechin mixture derived from green tea (Wako) was used as a positive control [20]. The prepared final suspensions were incubated at $37^{\circ} \mathrm{C}$ with constant shaking (100 strokes/min) for $3 \mathrm{~h}$ [20]; next, the size distribution and mean fat emulsion droplet sizes were measured using a particle size analyzer (Microtrac ${ }^{\bullet}$ MT3000II, Nikkiso Co., Ltd, Tokyo, Japan). Suspensions of the five bacterial strains (LG2055, LG1131T, LG0317, LB1002T and ST19258T) were prepared at a final concentration of $100 \mu \mathrm{g} / \mathrm{ml}$.

\section{Human study \\ Subjects}

Thirty healthy adults (12 men and 18 women) between $27-69$ years of age were enrolled in the study. Subjects with a frequency of defecation less than five days per week and severe internal organ disorders, including coronary heart disease, respiratory impairment, endocrinopathy, or alimentary allergy were excluded. None of the subjects consumed special health-promoting foods, took medications known to alter lipid metabolism, or regularly ingested fermented milk.

\section{Study design}

The study was performed as a double-blind, randomized, placebo-controlled clinical trial, according to the guidelines established in the Declaration of Helsinki. All procedures involving human subjects were approved by the institutional review board of the Miyawaki Orthopedic Clinic (Eniwa City, Hokkaido, Japan) prior to initiation of the study. All subjects provided written informed consent prior to study participation. The study was conducted from January 2014 to February 2014 by a contract research organization, New Drug Research Center, Inc. (Minato-ku, Tokyo, Japan). The clinical trial was registered at the University Hospital Medical Information Network Clinical Trials (No. UMIN000015772).

\section{Preparation of the test fermented milk}

Two types of fermented milk (FM) were prepared: the active FM containing LG2055 and the control FM lacking LG2055. The active FM was prepared using lactic acid bacteria starter cultures (Streptococcus thermophilus and Lactobacillus delbrueckii subsp. bulgaricus) commonly used for conventional yogurt production and viable LG2055 cells. An FM mixture consisting of approximately $11 \%$ skim milk powder with a small amount of flavor, agar, and sucralose as a non-caloric artificial sweetener was inoculated with the yogurt starter cultures and LG2055 cells, then cultured at $40^{\circ} \mathrm{C}$ for $3.5-4 \mathrm{~h}$. On the initial day, the viable cell count of LG2055 was approximately $5 \times 10^{9} \mathrm{CFU} / 100 \mathrm{~g}$ of FM. The control FM was prepared in the same manner, except that LG2055 cells 
were excluded. These FM preparations were equivalent in energy (146.4 kJ), protein ( $3.7 \mathrm{~g})$, fat $(0.1 \mathrm{~g})$, carbohydrate $(4.9 \mathrm{~g})$, and sodium content $(40 \mathrm{mg})$ per $100 \mathrm{~g}$ and were indistinguishable in taste. The test FM preparations were kept in cold storage and delivered weekly.

\section{Study schedule and protocol}

The study length was fourteen days, comprising a sevenday pre-observational period followed by a seven-day FM intake period. Subjects were randomized into the control and active FM groups. To equalize energy and fat intakes, subjects were provided the same diets throughout the entire study period (14 days), consisting of different menus at each meal, each day of the study. Energy intake was set at approximately 8368 and $9623.2 \mathrm{~kJ}$ per day in women and men, respectively; the amount of fat intake was set at approximately 70 and $85 \mathrm{~g}$ per day (31.5 and 33.3 percent energy) in women and men, respectively. After the seven-day preobservational period, both groups started consuming the test FM for seven days. The subjects consumed FM at $100 \mathrm{~g} / \mathrm{d}$ with meals (at either breakfast, lunch or dinner) and they were asked to maintain their normal lifestyle habits, including exercise routines. All subjects maintained a detailed dietary record during the entire study period. Fecal samples were collected during the final three days of the pre-observational (days 5-7) and FM intake periods (days 12-14). Fecal samples were weighed and immediately frozen for storage. Body weight, body fat percentage, blood pressure, pulse rate, and fasting blood parameters (triacylglycerol, total cholesterol, high density lipoprotein cholesterol, NEFA, glucose, total protein, aspartate aminotransferase, alanine aminotransferase, alkaline phosphatase, and gamma-glutamyl transpeptidase) were determined at the beginning of the test period (day 1) and the day after the end of the experimental period (day 15). Physician interviews were also performed at each time point (day 1 and 15). Information regarding subjective symptoms such as headache, nausea, and abdominal pain were obtained through a physician interview at each time point (day 1 and 15).

\section{Measurement of fecal fat concentration}

Fecal fat level was determined according to the methods described by van de Kamer et al. [21].

\section{Physical characteristics and blood sample analyses}

Blood analyses were performed by SRL, Inc. (Shinjuku-ku, Tokyo, Japan). The blood samples were centrifuged at $188 \times g$ for $10 \mathrm{~min}$ at $4^{\circ} \mathrm{C}$, and the supernatant was stored at a temperature below $-30^{\circ} \mathrm{C}$ until analysis. The concentration of serum NEFA was measured using a biochemical autoanalyzer JCA-BM6010 (JEOL Ltd., Tokyo, Japan). The concentrations of other serum parameters (triacylglycerol, total cholesterol, high density lipoprotein cholesterol, glucose, total protein, aspartate aminotransferase, alanine aminotransferase, alkaline phosphatase, and gamma-glutamyl transpeptidase) were measured using a biochemical autoanalyzer AY5400 (Beckman Coulter Inc., CA, USA).

\section{Statistical analysis}

All in vitro experiments were performed three times and data were expressed as means with standard deviation (SD). The Tukey-Kramer post-hoc test was used for multiple comparisons between strains. In the human study, the differences between the pre- and post-FM intake periods, and the differences between the control and active FM groups, were evaluated using Student's paired $t$-test and Student's unpaired $t$-test, respectively. A $P$ value $<0.05$ was considered statistically significant.

\section{Results}

Effects of LG2055 on pancreatic lipase activity in vitro

We first examined the effect of LG2055 on pancreatic lipase activity using a fat emulsion as a substrate in vitro (Figure 1). The lipase inhibitor orlistat [13] strongly inhibited lipase activity in a dose-dependent manner (0.001$1 \mu \mathrm{g} / \mathrm{ml}) ;$ LG2055 also suppressed lipase activity in a dosedependent manner $(1-100 \mu \mathrm{g} / \mathrm{ml}$ ) (Figure 1A). All the examined lactic acid bacterial species and strains significantly suppressed lipase activity at a final concentration of $100 \mu \mathrm{g} / \mathrm{ml}$ compared with the control lacking bacteria. Furthermore, LG2055 strongly suppressed lipase activity compared with the other four strains (LG1131T, LG0317, LB1002T, and ST19258T) (Figure 1B).

When lipase activity was measured using 4-MUO as a substrate (Figure 2), orlistat was strongly inhibitory, whereas LG2055 had no effect at concentrations ranging from 1-100 $\mu \mathrm{g} / \mathrm{ml}$. Thus, LG2055 did not suppress lipase activity using 4-MUO as a substrate. The other four strains (LG1131T, LG0317, LB1002T, and ST19258T) did not also suppress the lipase activity using 4-MUO (data not shown).

\section{Effects of LG2055 on fat emulsion droplet size in vitro}

The average fat emulsion droplet size remained mostly constant following incubation for $3 \mathrm{~h}$ in the absence of LG2055 (shortly after preparation: $2.00 \pm 0.41 \mu \mathrm{m}$, after incubation: $2.21 \pm 0.87 \mu \mathrm{m}$ ), indicating that the fat emulsion preparation was stable for a minimum of $3 \mathrm{~h}$.

The catechin mixture $(1000 \mu \mathrm{g} / \mathrm{ml})$ increased fat emulsion droplet size, as previously reported [20]. LG2055 significantly modified droplet size distribution at the same concentration as the catechin mixture (Figure 3A). Enlargement of the fat emulsion by LG2055 was also observed in phase-contrast micrographs (Figure 3B). 
A

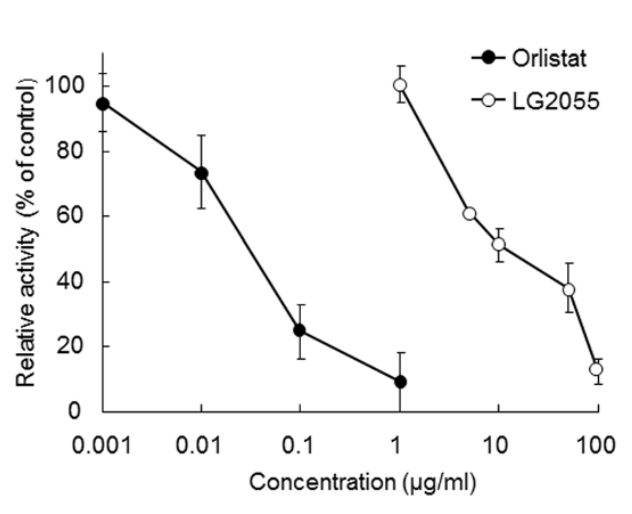

B

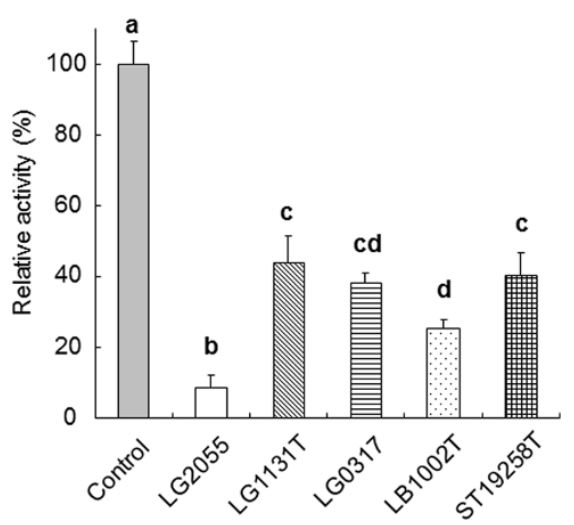

Figure 1 Suppressive effect of lactic acid bacteria on pancreatic lipase-mediated hydrolysis of triolein in an emulsion. Substrate suspensions (fat emulsion) were incubated with pancreatic lipase $\left(200 \mathrm{U} / \mathrm{ml}\right.$ ) and lactic acid bacterial cells or orlistat for $30 \mathrm{~min}$ at $37^{\circ} \mathrm{C}$. After boiling for 2 min, released fatty acids were quantified. The suppressive activity was calculated for fatty acid production, in which the activity in the absence of sample was represented as 100\%. (A) Lactobacillus gasseri SBT2055 (LG2055) suppressed the release of fatty acids from fat emulsion in a dose-dependent manner. (B) Comparison of the capacity of various lactic acid bacteria in suppressing fatty acid release. Each of the five bacterial strains (LG2055, Lactobacillus gasseri SBT0317 (LG0317), Lactobacillus gasseri JCM1131 (LG1131T), Lactobacillus delbrueckii subsp. bulgaricus JCM1002 ${ }^{\top}$ (LB1002T), and Streptococcus thermophilus ATCC19258 ${ }^{\top}$ (ST19258T)) was added to the lipase reaction solution at $100 \mu \mathrm{g} / \mathrm{ml}$. Values are means with standard deviations for triplicate experiments. Statistical differences between the strains were analyzed using the Tukey-Kramer post-hoc test and significant differences $(P<0.05)$ are indicated using different letters.

LG2055 dose-dependently increased the average fat emulsion droplet size at concentrations ranging from 1$100 \mu \mathrm{g} / \mathrm{ml}$ (Figure 4A). All five strains of lactic acid bacteria $(100 \mu \mathrm{g} / \mathrm{ml})$ significantly increased the average fat emulsion droplet size compared with the control lacking bacteria. In addition, LG2055 significantly increased droplet size compared with the other strains (Figure 4B).

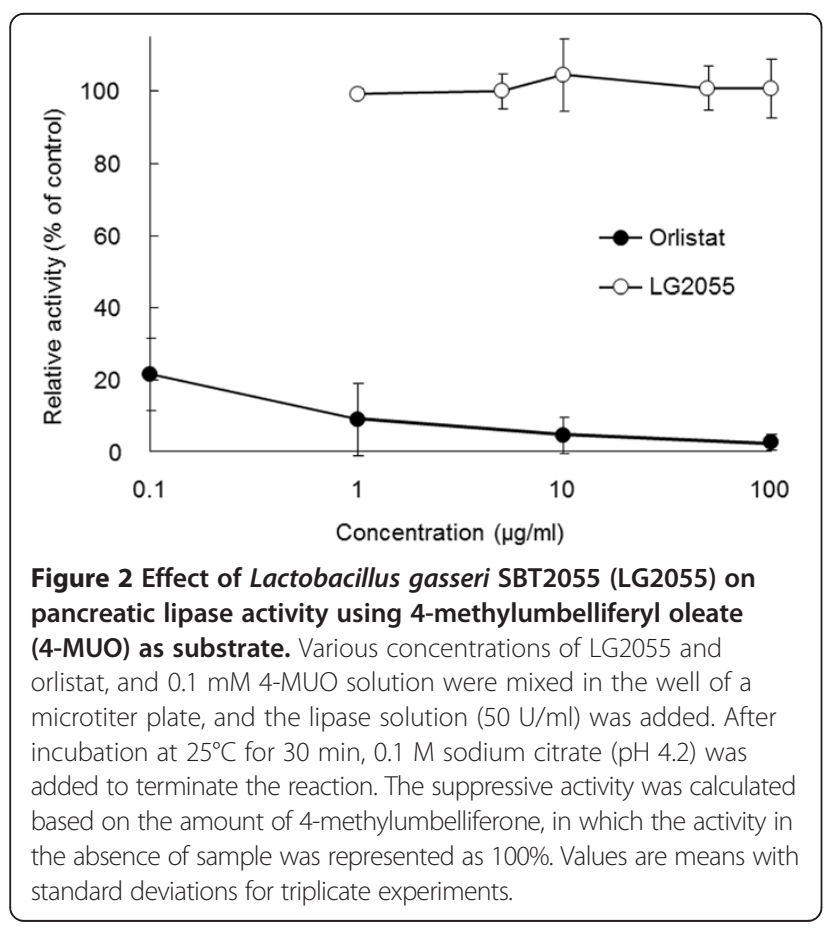

\section{Clinical study of fecal fat excretion}

The physical and biochemical blood characteristics of the subjects were not significantly different between groups (Table 1). No subject dropped out during the study.

The effects of the control and active FM preparations on physical and biochemical blood parameters are listed in Table 2. Body weight, body mass index (BMI), and total cholesterol levels significantly decreased similarly in both groups; alanine aminotransferase (ALT) and gamma-glutamyl transpeptidase $(\gamma-G T P)$ significantly decreased in the control FM group, and pulse rate significantly decreased in the active FM group but remained within the normal range. Based on daily records and physician interviews, no irregularities in daily life or adverse events related to FM consumption were observed during the study period, (data not shown).

There were no statistically significant differences in wet weight of feces between groups $(270.1 \pm 159.0 \mathrm{~g}$ in control FM group vs. $336.8 \pm 116.9 \mathrm{~g}$ in active FM group before FM intake; $264.9 \pm 151.0 \mathrm{~g}$ in control FM group vs. $304.4 \pm 128.7 \mathrm{~g}$ in active FM group after FM intake). After FM intake, fecal fat levels were significantly increased compared with those observed before FM intake in the active FM group, whereas no increase was observed in the control FM group (Figure 5A). The amount of change between pre- and post-FM intake periods increased in the active FM group compared with the control FM group, although the difference was not statistically significant $(P=0.086)$. 


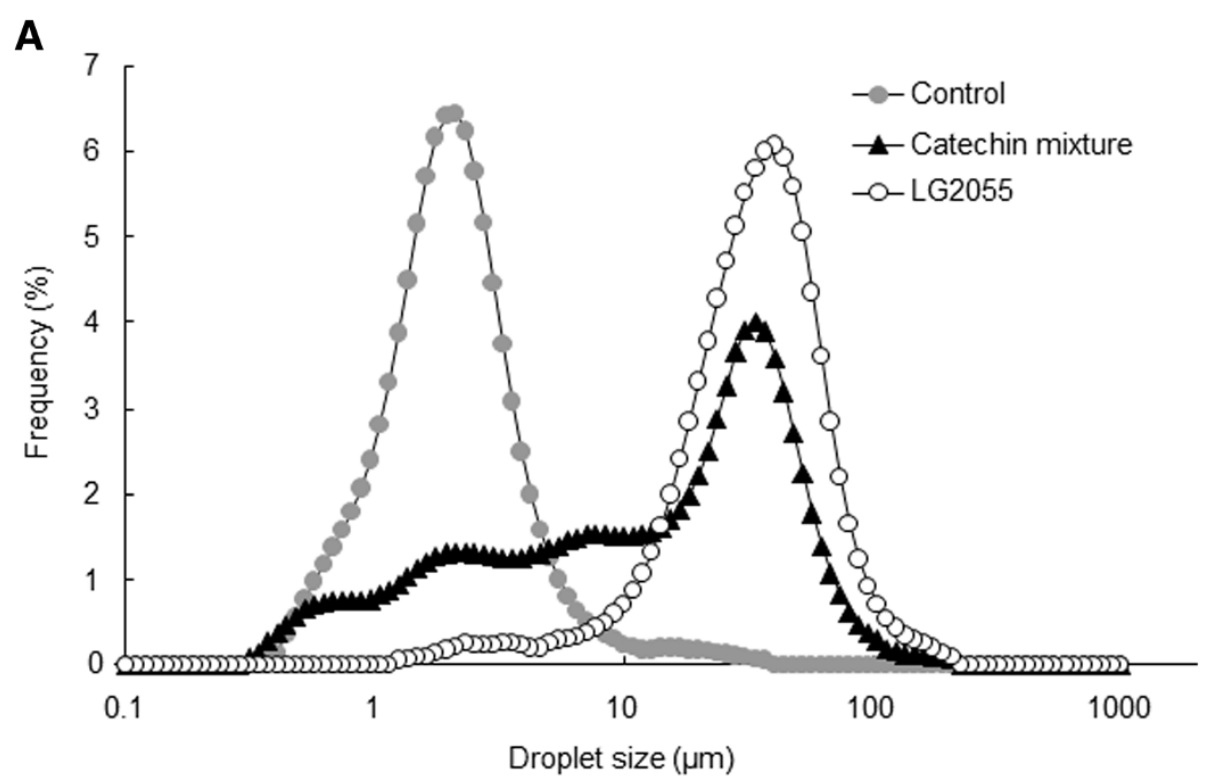

B

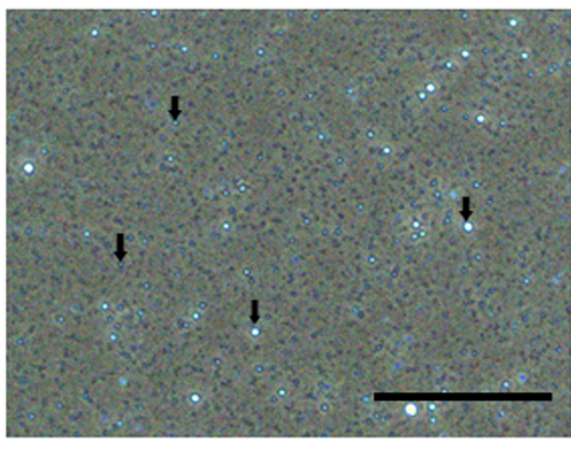

Control

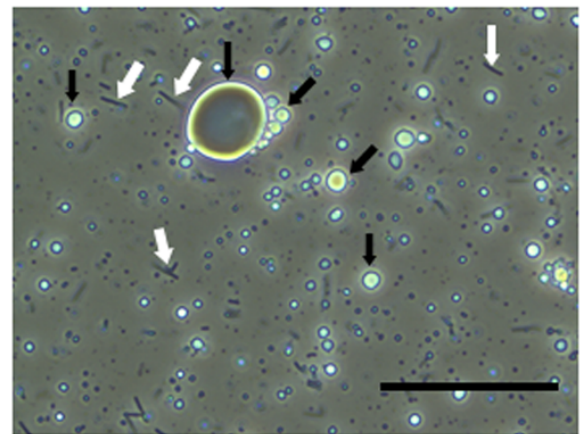

LG2055

Figure 3 Effect of Lactobacillus gasseri SBT2055 (LG2055) on fat emulsion droplet size in vitro. LG2055 or a catechin mixture was added to the fat emulsion preparation. The suspension was incubated at $37^{\circ} \mathrm{C}$ with constant shaking for $3 \mathrm{~h}$ and the size distribution of fat emulsion was measured using a particle size analyzer. No sample (only TES buffer) was added to the negative control and the catechin mixture was used as a positive control. (A) Changes in emulsion droplet size distribution after incubation with $1000 \mu \mathrm{g} / \mathrm{ml}$ of LG2055 or catechin mixture. The distributions are an average of three determinations. (B) Phase-contrast micrographs (scale bar: $50 \mu \mathrm{m}$ ). Black and white arrows indicate the representative fat emulsions and LG2055 cells, respectively.

\section{Discussion}

The suppression of pancreatic lipase-mediated fat hydrolysis is an effective strategy for prevention of obesity and hyperlipidemia. Pancreatic lipase inhibitors such as orlistat are currently used clinically as pharmaceutical agents [22]. In addition, it has been reported that dietary compounds, including chitosan [17], saponin [23], and oolong tea polyphenols [19], inhibit pancreatic lipase, consequently suppressing dietary lipid absorption. In this study, we observed that LG2055, a probiotic bacterium displaying anti-obesity properties, suppressed fatty acid release from fat emulsion in vitro (Figure 1A). Moreover, it was reported that LG2055 suppresses lipid absorption in the small intestine in vivo [4]. Taken together, these findings suggest that suppression of pancreatic lipase-mediated fat hydrolysis could represent a mechanism by which LG2055 mediates suppression of lipid absorption. Consistent with our findings, Matsumura [24] and Zhou et al. [25] reported that certain strains of Lactobacillus inhibit pancreatic lipase. The present study further demonstrated that all five examined lactic acid bacterial strains, including LG2055, had an ability to suppress lipase activity (fatty acid release from fat emulsion) to a certain extent (Figure 1B); however, LG2055 significantly suppressed fatty acid release from fat emulsion compared with the other strains, indicating a more potent effect. These results suggest that many strains of lactic acid bacteria likely have the ability to suppress lipase activity. However, LG2055 could represent a specific strain with an increased potential for lipase suppression among lactic acid bacteria. 

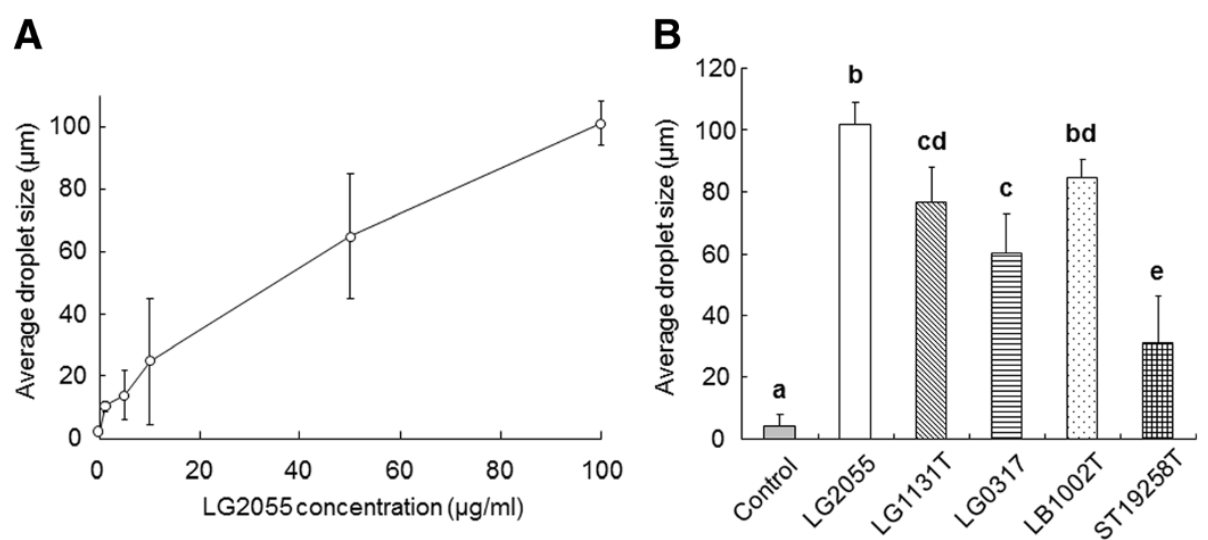

Figure 4 Increased effects of Lactobacillus gasseri SBT2055 (LG2055) on fat emulsion droplet size. (A) The dose-dependency of LG2055 on the average fat emulsion droplet size. (B) Comparison among lactic acid bacteria at $100 \mathrm{\mu g} / \mathrm{ml}$. Fat emulsion droplet size was measured in the same manner as described in Figure 3. Values are means with standard deviations for triplicate experiments. Statistical differences between the strains were analyzed using the Tukey-Kramer post-hoc test and significant differences $(P<0.05)$ are indicated using different letters.

The suppression of fat hydrolysis is mainly classified into two types: the first is suppression mediated by direct interaction between the inhibitor and enzyme, whereas the second is associated with modification of fat emulsion properties [26,27]. If LG2055 directly inhibits lipase-mediated hydrolysis, its suppressive effect should be observed when using a synthetic substrate such as 4MUO. We observed that orlistat inhibited lipase activity using 4-MUO as a substrate, consistent with results using fat emulsion. Conversely, LG2055 was not inhibitory (Figure 2), indicating that LG2055 does not directly inhibit pancreatic lipase. We also confirmed that all four

Table 1 Baseline characteristics of the study subjects

\begin{tabular}{|c|c|c|}
\hline Parameters & Control & Active \\
\hline Number of subjects & 15 & 15 \\
\hline \multicolumn{3}{|l|}{ Gender } \\
\hline Male & 6 & 6 \\
\hline Female & 9 & 9 \\
\hline Age (years) & $40.1 \pm 9.5$ & $42.6 \pm 10.8$ \\
\hline Height (cm) & $162.2 \pm \quad 6.4$ & $163.6 \pm 9.4$ \\
\hline Body weight (kg) & $57.3 \pm 6.4$ & $58.8 \pm 9.6$ \\
\hline Body fat percentage (\%) & $24.9 \pm 6.0$ & $25.4 \pm \quad 6.4$ \\
\hline BMI $\left(\mathrm{kg} / \mathrm{m}^{2}\right)$ & $21.8 \pm 2.5$ & $21.9 \pm 2.8$ \\
\hline Systolic blood pressure (mm Hg) & $116.6 \pm 13.4$ & $120.9 \pm 15.5$ \\
\hline Diastolic blood pressure (mm Hg) & $69.0 \pm 9.4$ & $77.0 \pm 13.0$ \\
\hline Pulse rate (beats/min) & $67.2 \pm 10.3$ & $69.1 \pm 7.3$ \\
\hline Triacylglycerol (mg/dl) & $65.1 \pm 22.0$ & $68.0 \pm 30.1$ \\
\hline Total cholesterol (mg/dl) & $197.1 \pm 24.7$ & $203.7 \pm 31.0$ \\
\hline Non-esterified fatty acid $(\mu \mathrm{Eq} / \mathrm{l})$ & $398.5 \pm 169.5$ & $393.3 \pm 173.7$ \\
\hline Glucose (mg/dl) & $82.4 \pm 6.6$ & $86.3 \pm 5.0$ \\
\hline
\end{tabular}

Values are means \pm SD. tested strains (LG1131T, LG0317, LB1002T, and ST19258T) did not inhibit lipase activity using 4-MUO as a substrate (data not shown). These results imply that LG2055 acts on the fat emulsion of a substrate rather than directly on the enzyme, leading to suppression of lipase-mediated hydrolysis. Fat emulsion interface properties, namely droplet size and specific surface area, control lipase-mediated fat emulsion hydrolysis $[14,28,29]$. Therefore, we examined whether LG2055 modifies fat emulsion properties by measuring fat emulsion droplet size upon mixing with LG2055. LG2055 promoted fat emulsion enlargement (Figure 3B) and dose-dependently increased fat emulsion droplet size (Figure 4A). An increase in droplet size is known to be associated with a decrease in specific surface area, resulting in delayed lipase-mediated hydrolysis. It has been reported that green tea extract [15], catechin mixture [20], and green coffee bean extract [20] increase fat emulsion droplet size, resulting in suppression of lipid absorption. However, there are no reports evaluating the effect of lactic acid bacteria on fat emulsion droplet size. Matsumura [24] and Zhou et al. [25] demonstrated that certain Lactobacillus strains inhibited pancreatic lipase activity but did not examine the mechanisms involved. To our knowledge, this is the first report describing an enlargement of fat emulsion by probiotics. With the exception of LG2055, the four tested strains also promoted an increase in fat emulsion droplet size (Figure 4B). However, LG2055 significantly increased droplet size compared with the other strains. These results are consistent with the strong suppressive effects of LG2055 on lipasemediated fat hydrolysis.

It remains unclear how LG2055 increases fat emulsion droplet size. One possibility is it that an interaction between LG2055 and bile acids (e.g. taurocholic acid) 
Table 2 Initial (day 1 ) and final (day 15) values of physical characteristics and biochemical blood parameters $(n=15)$

\begin{tabular}{|c|c|c|c|c|}
\hline \multirow{3}{*}{$\begin{array}{l}\text { Parameters } \\
\text { Body weight (kg) }\end{array}$} & \multicolumn{2}{|c|}{ Control } & \multicolumn{2}{|c|}{ Active } \\
\hline & day 1 & day 15 & day 1 & day 15 \\
\hline & $57.4 \pm \quad 6.5$ & $55.8 \pm 6.0^{* *}$ & $58.4 \pm 9.4$ & $57.2 \pm 9.2^{* *}$ \\
\hline Body fat percentage (\%) & $24.2 \pm 6.4$ & $24.2 \pm \quad 5.6$ & $25.1 \pm \quad 6.6$ & $25.1 \pm \quad 6.9$ \\
\hline BMI $\left(\mathrm{kg} / \mathrm{m}^{2}\right)$ & $21.9 \pm 2.6$ & $21.3 \pm 2.4^{* *}$ & $21.8 \pm \quad 2.8$ & $21.4 \pm \quad 2.9^{* *}$ \\
\hline Systolic blood pressure (mm Hg) & $117.3 \pm 15.5$ & $117.9 \pm 13.6$ & $115.9 \pm 14.4$ & $118.6 \pm 15.2$ \\
\hline Diastolic blood pressure (mm Hg) & $68.7 \pm 10.1$ & $69.1 \pm 10.4$ & $71.9 \pm 11.2$ & $73.0 \pm 11.6$ \\
\hline Pulse rate (beats/min) & $67.0 \pm 5.9$ & $65.9 \pm 10.6$ & $71.9 \pm 9.0$ & $67.0 \pm 8.3^{*}$ \\
\hline Triacylglycerol (mg/dl) & $67.5 \pm 33.5$ & $59.5 \pm 21.3$ & $67.2 \pm 37.2$ & $66.5 \pm 32.3$ \\
\hline Total cholesterol (mg/dl) & $188.5 \pm 17.9$ & $176.1 \pm 27.4^{*}$ & $192.7 \pm 35.4$ & $179.7 \pm 28.0^{*}$ \\
\hline HDL cholesterol (mg/dl) & $68.1 \pm 10.3$ & $68.7 \pm 11.1$ & $66.5 \pm 11.8$ & $65.6 \pm 11.5$ \\
\hline Non-esterified fatty acid $(\mu \mathrm{Eq} / \mathrm{l})$ & $331.8 \pm 105.5$ & $372.1 \pm 135.1$ & $413.1 \pm 223.8$ & $462.9 \pm 169.2$ \\
\hline Glucose (mg/dl) & $85.7 \pm 10.0$ & $82.5 \pm \quad 5.8$ & $85.3 \pm \quad 6.0$ & $84.0 \pm \quad 6.2$ \\
\hline Total protein (g/dl) & $7.4 \pm \quad 0.3$ & $7.4 \pm \quad 0.5$ & $7.5 \pm \quad 0.4$ & $7.5 \pm \quad 0.4$ \\
\hline AST (U/l) & $19.8 \pm \quad 4.6$ & $19.1 \pm$ & $19.4 \pm$ & $19.1 \pm \quad 4.6$ \\
\hline $\operatorname{ALT}(\mathrm{U} / \mathrm{l})$ & $20.3 \pm 11.6$ & $18.2 \pm 9.4^{*}$ & $15.7 \pm \quad 5.6$ & $14.3 \pm \quad 5.0$ \\
\hline $\operatorname{ALP}(\mathrm{U} / \mathrm{l})$ & $185.4 \pm 35.1$ & $176.2 \pm 35.7$ & $185.1 \pm 38.6$ & $188.3 \pm 30.4$ \\
\hline Y-GTP (U/I) & $23.2 \pm 14.1$ & $18.6 \pm 9.1^{*}$ & $21.3 \pm 10.5$ & $18.5 \pm \quad 8.8$ \\
\hline
\end{tabular}

Abbreviations: $H D L$ high density lipoprotein, AST aspartate aminotransferase, $A L T$ alanine aminotransferase, ALP alkaline phosphatase, $\gamma$-GTP gamma-glutamyl transpeptidase.

Values are means \pm SD

Significantly different from the initial values within the group; ${ }^{*} P<0.05,{ }^{* *} P<0.01$

could contribute to the modification of fat emulsion properties. Interestingly, it was reported that certain Lactobacillus strains have an ability to bind and deconjugate taurocholic acid [30]. LG2055 also has an ability to deconjugate taurocholic acid [16]. Bile acids have amphipathic properties and are critical for the emulsification of dietary lipids in the intestine [31]. As our preliminary study demonstrated, LG2055 did not increase fat emulsion droplet size without inclusion of taurocholic acid prepared by the method reported by Shishikura et al. [15]
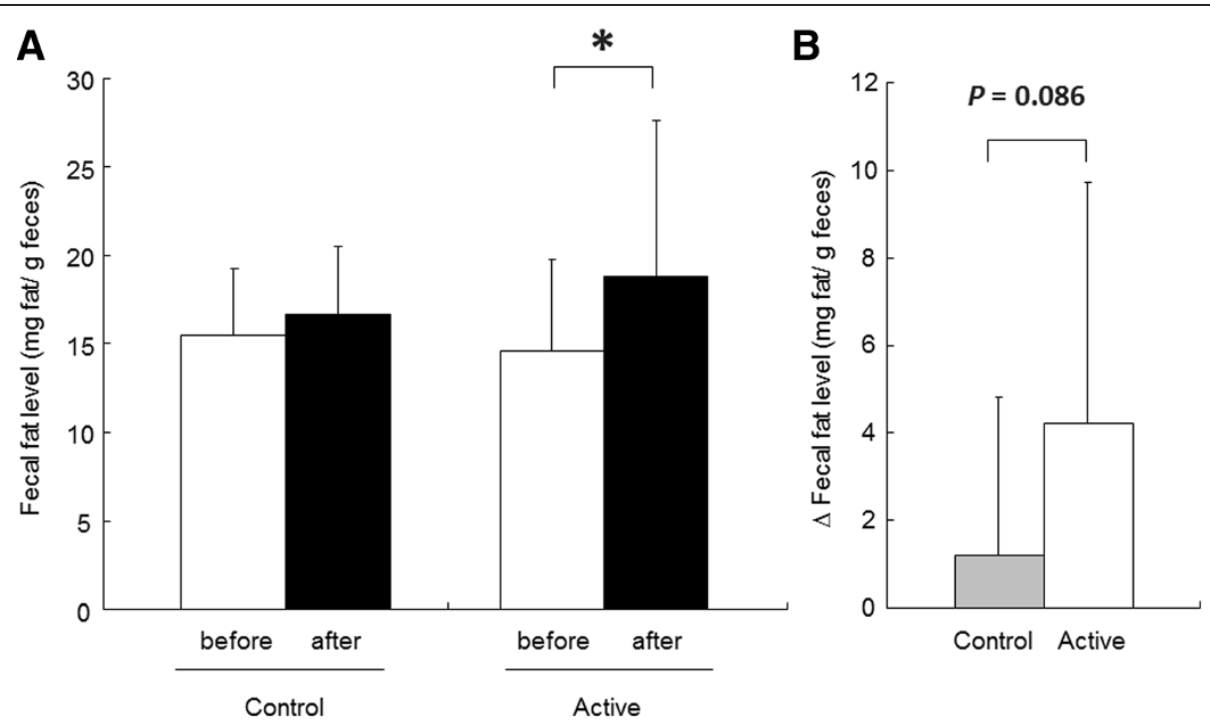

Figure 5 Effect of intake of FM containing Lactobacillus gasseri SBT2055 (LG2055) on fecal fat excretion in humans. (A) Fecal samples were collected during the final three days of the pre-observational period (before) and FM intake period (after), and fecal fat levels were determined. (B) The amount of change in the fecal fat levels in the control and active FM groups. Values were calculated by subtracting fecal fat levels at pre-observational period from those at FM intake period. Values are means with standard deviations. Statistical analysis was performed using paired Student's t-test (A) and unpaired Student's $t$-test (B). An asterisk indicates a significant difference $(P<0.05)$. 
(data not shown). LG2055 potentially interacts with bile acids and destabilizes the fat emulsion, resulting in its coalescence. Usman et al. previously reported that LG0317, a strain included in the present study, had a decreased capacity to deconjugate taurocholic acid compared with LG2055 [16]. In our study, LG0317 had a decreased effect on fat emulsion enlargement. These findings indicate the importance of the interaction between taurocholic acid and lactic acid bacteria for promotion of increased fat emulsion droplet size.

We recently reported that consumption of FM containing LG2055 decreased postprandial serum triacylglycerol and non-esterified fatty acid (NEFA) concentrations in peripheral blood after intake of oral fat-loading test meals compared with consumption of FM without LG2055 [10]. Although triacylglycerol and NEFA concentrations in peripheral blood are important biomarkers of fat absorption, they are affected by metabolism in organs such as the liver [32]. Thus, we evaluated fecal lipid excretion to assess more directly the effect of LG2055 on lipid absorption in humans. Our data show that consumption of FM containing LG2055 increased fecal fat excretion (Figure 5A/B). Given that LG2055 suppressed pancreatic lipase-mediated fat hydrolysis in vitro, its effects on fecal fat excretion are likely associated with suppression of lipase-mediated fat hydrolysis, resulting in decreased lipid absorption. In this study, we observed that BMI and total serum cholesterol concentration were significantly decreased, but remained within the normal range, after FM intake in both the control and active FM groups (Table 2). We consider that this is because energy intake by the subjects was moderately controlled, or beneficial effects of fermented milk on lipid metabolism [33] were taken. Consumption of LG2055 for seven days did not significantly influence body fat percentage. However, constant ingestion of LG2055 over a prolonged period could reveal more pronounced anti-obesity effects, as we previously demonstrated when LG2055 was consumed daily for 12 weeks [9].

\section{Conclusions}

In conclusion, our findings clearly demonstrate that LG2055 modifies the physicochemical properties of fat emulsion by increasing droplet size and suppressing fatty acid release from fat emulsion. These data provide a mechanism by which LG2055 mediates the suppression of lipid absorption and an increase in fecal fat excretion in humans.

\section{Abbreviations}

LG2055: Lactobacillus gasseri SBT2055; BMl: Body mass index; 4-MUO: 4methylumbelliferyl oleate; TES: N-tris (hydroxymethyl) methyl-2aminoethanesulfonic acid; LG0317: Lactobacillus gasseri SBT0317; LG1131T: Lactobacillus gasseri JCM1131'; LB1002T: Lactobacillus delbrueckii subsp. bulgaricus JCM1002'ं ST19258T: Streptococcus thermophilus ATCC19258'; FM: Fermented milk; NEFA: Non-esterified fatty acid.
Competing interests

The authors declare that they have no competing interests.

\section{Authors' contributions}

$\mathrm{AO}$ and FS wrote the manuscript. TK was responsible for the clinical study. YK and YK assisted with data analysis and interpretation. All authors read and approved the final manuscript.

\section{Acknowledgments}

We thank the study participants for their cooperation. We also thank $\mathrm{Mr}$. Shimpei Tomita (New Drug Research Center Inc., Tokyo, Japan), Dr. Ikuo Fukuhara (Fukuhara Clinic, Eniwa City, Hokkaido, Japan), and the staff members of these facilities that were involved in clinical study.

\section{Author details}

${ }^{1}$ Milk Science Research Institute, Megmilk Snow Brand Co. Ltd., 1-1-2 Minamidai, Kawagoe, Saitama 350-1165, Japan. ${ }^{2}$ Public Relations Department, Megmilk Snow Brand Co. Ltd., 13 Honshiocho, Shinjuku-ku, Tokyo 160-0003, Japan.

Received: 17 December 2014 Accepted: 5 March 2015

Published online: 20 March 2015

\section{References}

1. Seto Y, Kimura A, Akai Y, Fujiwara S. Distribution of the Lactobacillus acidophilus complex in human fecal specimens examined by restriction fragment length polymorphism of the $16 \mathrm{~S}$ rRNA genes. Biosci Microflora. 2003;22:75-83.

2. Takahashi H, Fujita T, Suzuki Y, Benno Y. Monitoring and survival of Lactobacillus gasseri SBT2055 in the human intestinal tract. Microbiol Immunol. 2006;50:867-70.

3. Fujiwara S, Seto Y, Kimura A, Hashiba H. Establishment of orallyadministered Lactobacillus gasseri SBT2055SR in the gastrointestinal tract of humans and its influence on intestinal microflora and metabolism. J Appl Microbiol. 2001;90:343-52.

4. Hamad EM, Sato M, Uzu K, Yoshida T, Higashi S, Kawakami H, et al. Milk fermented by Lactobacillus gasseri SBT2055 influences adipocyte size via inhibition of dietary fat absorption in Zucker rats. Br J Nutr. 2009;101:716-24.

5. Sato M, Uzu K, Yoshida T, Hamad EM, Kawakami H, Matsuyama H, et al. Effects of milk fermented by Lactobacillus gasseri SBT2055 on adipocyte size in rats. Br J Nutr. 2008;99:1013-7.

6. Kadooka Y, Ogawa A, Ikuyama K, Sato M. The probiotic Lactobacillus gasseri SBT2055 inhibits enlargement of visceral adipocytes and upregulation of serum soluble adhesion molecule (sICAM-1) in rats. Int Dairy J. 2011;21:623-7.

7. Miyoshi M, Ogawa A, Higurashi S, Kadooka Y. Anti-obesity effect of Lactobacillus gasseri SBT2055 accompanied by inhibition of pro-inflammatory gene expression in the visceral adipose tissue in diet-induced obese mice. Eur J Nutr. 2014;53:599-606.

8. Kadooka Y, Sato M, Imaizumi K, Ogawa A, Ikuyama K, Akai Y, et al. Regulation of abdominal adiposity by probiotics (Lactobacillus gasseri SBT2055) in adults with obese tendencies in a randomized controlled trial. Eur J Clin Nutr. 2010;64:636-43.

9. Kadooka Y, Sato M, Ogawa A, Miyoshi M, Uenishi H, Ogawa H, et al. Effect of Lactobacillus gasseri SBT2055 in fermented milk on abdominal adiposity in adults in a randomized controlled trial. Br J Nutr. 2013;110:1696-703.

10. Ogawa A, Kadooka Y, Kato K, Shirouchi B, Sato M. Lactobacillus gasseri SBT2055 reduces postprandial and fasting serum non-esterified fatty acid levels in Japanese hypertriacylglycerolemic subjects. Lipids Health Dis. 2014;13:36.

11. Lowe ME. Pancreatic triglyceride lipase and colipase: Insights into dietary fat digestion. Gastroenterology. 1994;107:1524-36

12. Tucci SA, Boyland EJ, Halford JC. The role of lipid and carbohydrate digestive enzyme inhibitors in the management of obesity: a review of current and emerging therapeutic agents. Diabetes Metab Syndr Obes. 2010:3:125-43.

13. Hvizdos KM, Markham A. Orlistat: A review of its use in the management of obesity. Drugs. 1999;58:743-60.

14. Armand M, Pasquier B, André M, Borel P, Senft M, Peyrot J, et al. Digestion and absorption of 2 fat emulsions with different droplet sizes in the human digestive tract. Am J Clin Nutr. 1999;70:1096-106. 
15. Shishikura Y, Khokhar S, Murray BS. Effects of tea polyphenols on emulsification of olive oil in a small intestine model system. J Agric Food Chem. 2006;54:1906-13.

16. Usman, Hosono A. Bile tolerance, taurocholate deconjugation, and binding of cholesterol by Lactobacillus gasseri strains. J Dairy Sci. 1999;82:243-48.

17. Han LK, Kimura Y, Okuda H. Reduction in fat strage during chitin-chitosan treatment in mice fed a high-fat diet. Int J Obes. 1999;23:174-9.

18. Kurihara H, Asami S, Shibata H, Fukami H, Tanaka T. Hypolipemic effect of Cyclocarya paliurus (Batal) Iljinskaja in lipid-loaded mice. Biol Pharm Bull. 2003;26:383-5

19. Nakai M, Fukui $Y$, Asami S, Toyoda-Ono Y, Iwashita T, Shibata H, et al. Inhibitory effects of oolong tea polyphenols on pancreatic lipase in vitro. J Agric Food Chem. 2005;53:4593-8.

20. Narita Y, Iwai K, Fukunaga T, Nakagiri O. Inhibitory activity of chlorogenic acids in decaffeinated green coffee beans against porcine pancreas lipase and effect of a decaffeinated green coffee bean extract on an emulsion of olive oil. Biosci Biotechnol Biochem. 2012:76:2329-31.

21. van de Kamer JH, Ten Bokkel HH, Weyers HA. Rapid method for the determination of fat in feces. J Biol Chem. 1949;177:347-55.

22. Hill JO, Hauptman J, Anderson JW, Fujioka K, O'Neil PM, Smith DK, et al. Orlistat, a lipase inhibitor, for weight maintenance after conventional dieting: A 1-y study. Am J Clin Nutr. 1999;69:1108-16.

23. Oishi Y, Sakamoto T, Udagawa H, Taniguchi H, Kobayashi-Hattori K, Ozawa $Y$, et al. Inhibition of increases in blood glucose and serum neutral fat by Momordica charantia saponin fraction. Biosci Biotechnol Biochem. 2007:71:735-40.

24. Matsumura A. Inhibitory effects of probiotics on pancreatic lipase. J Intest Microbiol (in Japanese). 2010;24:287-92.

25. Zhou Y, Inoue N, Ozawa R, Maekawa T, Izumo T, Kitagawa Y, et al. Effects of heat-killed Lactobacillus pentosus S-PT84 on postprandial hypertriacylglycerolemia in rats. Biosci Biotechnol Biochem. 2013;77:591-4.

26. Satouchi K, Mori T, Matsushita S. Characterization of inhibitor protein for lipase in soybean seeds. Agric Biol Chem. 1974;38:97-101.

27. Satouchi K, Matsushita S. Purification and properties of a lipase inhibiting protein from soybean cotyledons. Agric Biol Chem. 1976:40:889-97.

28. Armand M, Borel P, Ythier P, Dutot G, Melin C, Senft M, et al. Effects of droplet size, triacylglycerol composition, and calcium on the hydrolysis of complex emulsions by pancreatic lipase: an in vitro study. J Nutr Biochem. 1992:3:333-41.

29. Borel $P$, Armand M, Ythier P, Dutot G, Melin C, Senft M, et al. Hydrolysis of emulsions with different triglycerides and droplet sizes by gastric lipase in vitro. Effect on pancreatic lipase activity. J Nutr Biochem. 1994;5:124-33.

30. Hashimoto H, Kawase M, Hosoda M, Fang HE, Morita H, Hosono A. Binding deconjugation and oxidation of taurocholic acid with lactobacilli cells. Milchwissenschaft. 2000;55:316-9

31. Fillery-Travis AJ, Foster LH, Robins MM. Stability of emulsions stabilised by two physiological surfactants: L-alpha-phosphatidylcholine and sodium taurocholate. Biophys Chem. 1995:54:253-60.

32. Lambert JE, Parks EJ. Postprandial metabolism of meal triglyceride in humans. Biochim Biophys Acta. 1821;2012:721-6.

33. Crichton GE, Alkerwi A. Dairy food intake is positively associated with cardiovascular health: findings from Observation of Cardiovascular Risk Factors in Luxembourg study. Nutr Res. 2014;34:1036-44.

\section{Submit your next manuscript to BioMed Central and take full advantage of:}

- Convenient online submission

- Thorough peer review

- No space constraints or color figure charges

- Immediate publication on acceptance

- Inclusion in PubMed, CAS, Scopus and Google Scholar

- Research which is freely available for redistribution 